\title{
Mortalidade infantil de indígenas e não indígenas nas microrregiões do Brasil
}

\author{
Infant mortality among indigenous and non-indigenous people in the Brazilian microregions \\ Mortalidad infantil de indígenas y no indígenas en las microrregiones de Brasil
}

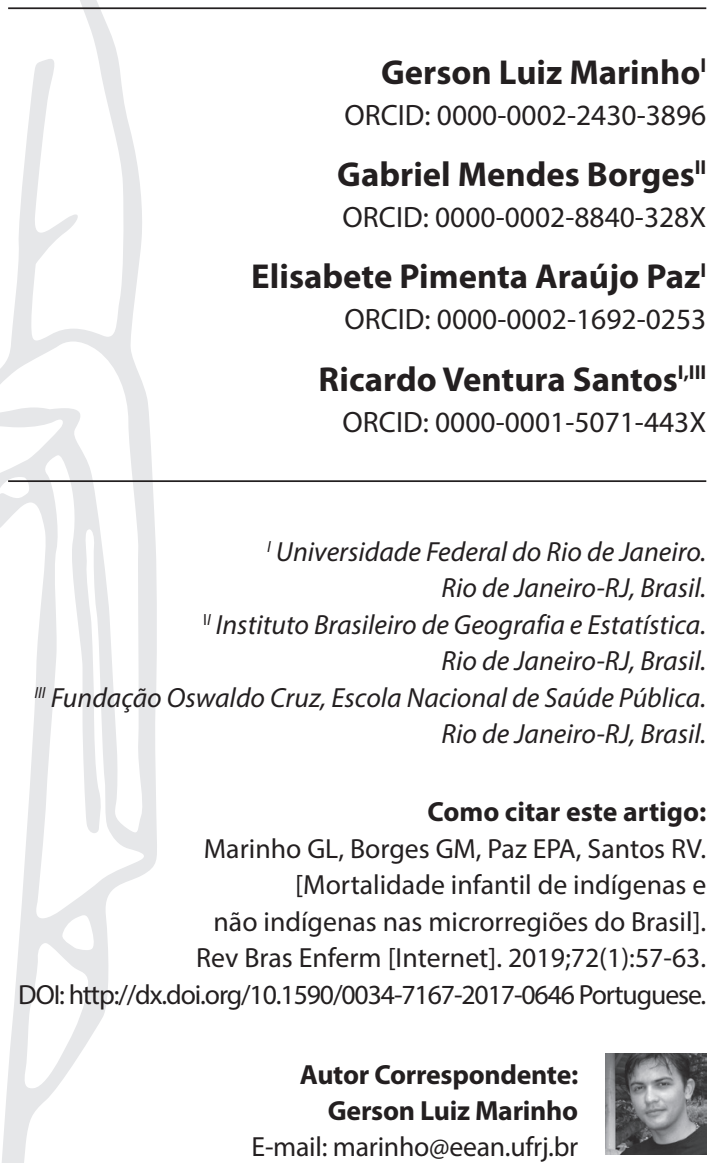

Submissão: 25-11-2017

Aprovação: 20-04-2018

\section{RESUMO}

Objetivo: Analisar a mortalidade infantil de indígenas e não indígenas nas microrregiões do Brasil. Método: Os dados são oriundos do Censo Demográfico 2010 e do Sistema de Informações sobre Mortalidade. Taxas e proporções caracterizaram a mortalidade infantil nas 558 microrregiões existentes em 2010. Resultados: No Brasil, crianças indígenas apresentaram elevados riscos de morrer antes de completarem um ano de idade (60\% maior em relação às não indígenas), sendo mais expressivo nas microrregiões com população indígena inferior a $1 \%$. A cada 10 óbitos infantis indígenas, sete eram crianças com mais de um mês de vida acometidas por doenças infecciosas. Conclusão: Os óbitos infantis indígenas são, de modo geral, evitáveis através da realização de intervenções no âmbito da Atenção Primária à Saúde. Há importantes diferenças nos níveis de mortalidade infantil entre indígenas e não indígenas, inclusive nos contextos geográficos onde se fazem presentes maiores contingentes de indígenas.

Descritores: Mortalidade Infantil; População Indígena; Raça e Saúde; Desigualdades em Saúde; Censos Demográficos.

\section{ABSTRACT}

Objective: To compare infant mortality rates of indigenous and non-indigenous children according to microregions in Brazil. Method: The study was based on data from the 2010 Population Census and from the Mortality Information System. Rates and proportions were calculated using data from 558 microregions. Results: Indigenous children presented a higher risk of dying before completing one year of age $(60 \%$ higher compared to non-indigenous), and rates were higher in microregions with $<1 \%$ of indigenous population. Seven out of 10 deaths were in children over one month of age and were mostly caused by infectious diseases. Conclusion: In general, indigenous children die mostly due to preventable causes. The study shows that there are important differences in the levels of infant mortality between indigenous and nonindigenous children in Brazil, even in geographical areas with increased presence of indigenous population.

Descriptors: Infant Mortality; Indigenous Population; Race and Health; Inequalities in Health; Population Censuses.

\section{RESUMEN}

Objetivo: Analizar la mortalidad infantil de indígenas y no indígenas en las microrregiones de Brasil. Método: Los datos provienen del Censo Demográfico del 2010 y del Sistema de Información acerca de la Mortalidad. Las tasas y proporciones caracterizan la mortalidad infantil en las 558 microrregiones existentes en el 2010. Resultados: Los niños indígenas presentaron elevado riesgo de morir antes de cumplir un año de edad (un $60 \%$ superior respecto a los no indígenas), y es más expresivo en las microrregiones con población indígena inferior al $1 \%$. Siete de cada 10 óbitos infantiles indígenas fueron de niños con más de un mes de vida, víctimas de enfermedades infecciosas. Conclusión: Los óbitos infantiles indígenas, de modo general, podrían ser evitados por intervenciones en el ámbito da Atención Primaria a la Salud. Los niveles de mortalidad entre los indígenas y no indígenas se mantienen incluso en contextos geográficos con los mayores contingentes de población indígena.

Descriptores: Mortalidad Infantil; Población Indígena; Raza y Salud; Desigualdades en Salud; Censos Demográficos. 


\section{INTRODUÇÃO}

A mortalidade de crianças menores de um ano de idade constitui um dos mais importantes marcadores das condições de vida, além de permitir avaliar o desempenho dos serviços de saúde ofertados a uma população ${ }^{(1-2)}$. Nas últimas décadas, vem sendo registrada importante diminuição das Taxas de Mortalidade Infantil (TMI) no Brasil como um todo, o que tem sido atribuído a um complexo conjunto de fatores sócio-demográficos e epidemiológicos ${ }^{(2)}$. Nas investigações em saúde pública, tem sido cada vez mais frequente a preocupação em destacar a magnitude das iniquidades em saúde sob uma perspectiva que considere a composição da população, segundo categorias de pertencimento racial e étnico ${ }^{(3-4)}$.

As poucas investigações acerca da mortalidade infantil entre os indígenas têm destacado que a redução das taxas para este segmento específico não acontece com a mesma velocidade e magnitude observadas para o restante da população brasileira ${ }^{(3,5-6)}$. De modo geral, os estudos sobre a dinâmica demográfica dos indígenas vêm se expandindo, mas as características da mortalidade infantil permanecem pouco conhecidas ${ }^{(7)}$.

No tocante às desigualdades em saúde, é importante avançar no sentido de se buscar investigar padrões, segundo cor ou raça, relacionando-os com estratificações geográficas específicas e relevantes. Para tal, no caso dos indígenas, os dados dos censos demográficos são particularmente importantes, dado que desde 1991 houve a inclusão da categoria "indígena" na pergunta sobre cor ou raça(8). Estratégias metodológicas que considerem unidades de análises alternativas são fundamentais para o campo da Saúde Indígena, uma vez que o subsistema específico para os povos indígenas se organiza operacionalmente, segundo divisões geográficas específicas, chamadas Distritos Sanitários Especiais Indígenas (DSEI) ${ }^{(9)}$.

\section{OBJETIVO}

Conduzir uma análise da mortalidade infantil de indígenas e não indígenas com base nos dados do Censo Demográfico 2010, com foco nas ocorrências observadas para as microrregiões geográficas, constituindo uma abordagem inédita no país. Além dos dados censitários, realizou-se análise das causas e dos componentes etários da mortalidade infantil a partir do Sistema de Informação sobre Mortalidade (SIM), enfocando o conjunto de microrregiões com os maiores contingentes de população indígena.

\section{MÉTODO}

\section{Aspectos éticos}

As análises deste estudo foram conduzidas através de dados disponibilizados por agências governamentais responsáveis pela geração de informações demográficas, socioeconômicas e epidemiológicas, representativas para o Brasil (Instituto Brasileiro de Geografia e Estatística - IBGE e Departamento de Informática do Ministério da Saúde - DATASUS). Para garantir o sigilo de informações individuais, os dados somente podem ser acessados segundo agrupamentos para os vários estratos geográficos. No caso do Censo 2010, os setores censitários (unidades espaciais com o mínimo de 800 moradores $^{(8)}$ ) representaram o menor nível geográfico de agrupamento dos dados. O conjunto de setores formavam bairros que, por sua vez, constituíam municípios, e assim sucessivamente. Já no sistema do Ministério da Saúde (SIM), o menor nível de agregação dos dados são os municípios brasileiros, que totalizavam 5.505 em 2010.

Em consonância com as normas sobre ética em pesquisas com seres humanos vigentes no Brasil, pesquisas realizadas com dados secundários e que mantenham o anonimato dos investigados não precisam ser submetidas à apreciação por Comitê de Ética em Pesquisa (Resolução nº 466/2012 CONEP). Além disso, o acesso aos dados públicos que foram coletados por órgãos governamentais também se ampara na Lei de Acesso à Informação (Lei nº 12.527/2011).

\section{Desenho, local do estudo e período}

Empregou-se o delineamento transversal, com análises sobre mortalidade infantil, representativas para todo o país e estratificações, segundo microrregiões existentes no ano de 2010 ( $N=558$ ).

Os dados são oriundos do Censo Demográfico 2010 realizado pelo Instituto Brasileiro de Geografia e Estatística (IBGE) e do Sistema de Informações sobre Mortalidade (SIM/DATASUS) (www. datasus.gov.br). As informações censitárias foram obtidas através do Banco Multidimensional de Estatísticas - BME/IBGE (www. bme.ibge.gov.br), ferramenta que disponibiliza informações das principais pesquisas nacionais conduzidas pelo Instituto, apresentando-as de modo agregado para diferentes estratos geográficos (grandes regiões, unidades da federação, microrregiões, etc.).

De acordo com o IBGE ${ }^{(10)}$ :

As microrregiões geográficas são conjunto de municípios contíguos, definidas como partes das mesorregiões que apresentam especificidades quanto à organização do espaço. Sua delimitação leva em conta, além das dimensões formadoras das mesorregiões, a vida de relações em nível local, pela possibilidade de atendimento às suas populações, por parte dos setores sociais básicos (...)

\section{População, critérios de inclusão e exclusão}

Foram selecionados os dados sobre óbitos de crianças menores de um ano de idade, coletados pelo Censo Demográfico 2010 e os notificados pelo SIM/DATASUS no mesmo ano. As análises foram conduzidas de modo a comparar ocorrências e magnitudes das mortes de crianças indígenas e não indígenas. Denominou-se como "não indígena" o conjunto dos óbitos registrados para as demais categorias de cor ou raça presentes no Censo 2010 e no SIM/DATASUS, quais sejam: branca, preta, amarela e parda.

A ocorrência de mortes de crianças menores de um ano foi considerada, em relação à população de crianças vivas com idade inferior a um ano residentes nos domicílios do Brasil, de acordo com o Censo 2010. As causas dos óbitos, segundo capítulos da

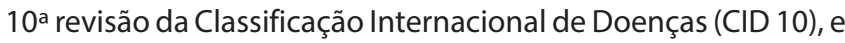
os componentes etários da mortalidade infantil foram descritos a partir do SIM/DATASUS em contextos geográficos específicos (microrregiões onde, pelo menos, $10 \%$ do total da população total era indígena, em 2010). 


\section{Protocolo do estudo}

No Censo 2010, a investigação da ocorrência de óbitos e o pertencimento de cor ou raça se deu através do Questionário Básico, cujas 37 questões foram aplicadas a todos os domicílios existentes no Brasil (Universo), tendo como período de referência os doze meses anteriores a 31 de julho de 2010. Uma vez que o censo não levantou dados acerca da classificação de cor ou raça dos falecidos, a estratégia analítica empregada neste estudo foi atribuir aos óbitos a categoria de cor/raça da pessoa identificada como responsável pelo domicílio. Tal metodologia vem sendo aplicada em estudos recentes realizados a partir de dados censitários ${ }^{(5,7)}$.

Quanto ao SIM/DATASUS, foram analisadas as causas de morte de crianças indígenas e não indígenas, segundo capítulos da Classificação Internacional de Doenças (CID-10), e ocorrência de mortes no período neonatal (precoce $=0-6$ dias e tardia $=7$ a 28 dias) e pós-neonatal (29 a 364 dias). Para tal, foram selecionados os óbitos ocorridos em um conjunto de nove microrregiões nas quais os indígenas representavam $10 \%$ ou mais da população total, tendo o período de 01 de janeiro a 31 de dezembro de 2010 como referência.

Os dados derivados do BME e do SIM/DATASUS foram armazenados em planilhas eletrônicas e analisados no programa SPSS versão 20.0 (IBM, 2012). Para a confecção do mapa de microrregiões, foi utilizado o programa TerraView (INPE, 2013).

\section{Análise dos resultados e estatística}

A ocorrência de mortes de crianças menores de um ano de idade foi analisada comparativamente entre indígenas e não indígenas através do cálculo de Taxas de Mortalidade Infantil (TMI) para as microrregiões do Brasil. Para os óbitos derivados do Censo 2010 (numeradores), foi aplicado o fator de correção de 1,20 (valor intermediário entre os fatores propostos para óbitos do sexo masculino, de 1,23 , e feminino, de 1,17$)^{(11)}$.

Em geral, os denominadores das TMI correspondem ao número de nascidos vivos ocorridos no mesmo período dos óbitos ${ }^{(12)}$, mas esse quesito foi investigado somente pela amostra do Censo 2010 (total de filhos tidos no ano anterior à entrevista), uma vez que compôs o conjunto de questões sobre fecundidade ${ }^{(10)}$. Ainda que disponíveis, essa informação tende a ser subnotificada nos censos demográficos, requerendo ajustes por meio de técnicas demográficas indiretas ${ }^{(13)}$. Para fins do presente estudo, as frequências de crianças menores de um ano captadas pelo censo foram adotadas como denominadores das taxas de mortalidade, corrigidas de acordo com o proposto por Wachter ${ }^{(12)}$ e Keytz \& Flieger ${ }^{(13)}$. Dessa forma, as Taxas de Mortalidade Infantil $\left(q_{1}\right)$ foram calculadas, segundo a fórmula:

onde: $\mathrm{n}=1, \mathrm{M}_{0} \quad \mathrm{e}_{1} \mathrm{a}_{0}=\left[0,07+1,7\left({ }_{1} \mathrm{M}_{0}\right)\right]$.

Os óbitos de menores de um ano contabilizados pelo Censo 2010 foram estratificados de acordo com a proporção de indígenas em relação à população total. Os resultados são apresentados, segundo os seguintes estratos: microrregiões com menos de 1,0\% da população total composta por indígenas (487 microrregiões); de $1,0 \%$ a $2,9 \%$ (36); de $3,0 \%$ a $4,9 \%$ (12); de $5,0 \%$ a $9,9 \%$ (14) e mais de $10,0 \%(9)$.

Decorrente do critério indicado acima, somente para as nove microrregiões com mais de $10 \%$ da população composta por indígenas foram calculadas razões das TMI entre indígenas e não indígenas. Do mesmo modo, para esse conjunto de microrregiões, foram comparadas a mortalidade proporcional de acordo com causas (Capítulos CID 10) e os componentes da mortalidade infantil (neonatal precoce, neonatal e pós-neonatal).

\section{RESULTADOS}

O Censo 2010 registrou 35.055 óbitos de crianças menores de um ano de idade, dos quais 470 foram de crianças classificadas como indígenas. As microrregiões com os mais expressivos volumes populacionais de indígenas estavam localizadas majoritariamente nas regiões Norte e Centro-Oeste, sobrepondo-se à Amazônia (Figura 1). Das 35 microrregiões onde, em cada uma, os indígenas representavam pelo menos $3,0 \%$ do total da população, 24 estavam no Norte e Centro-Oeste.

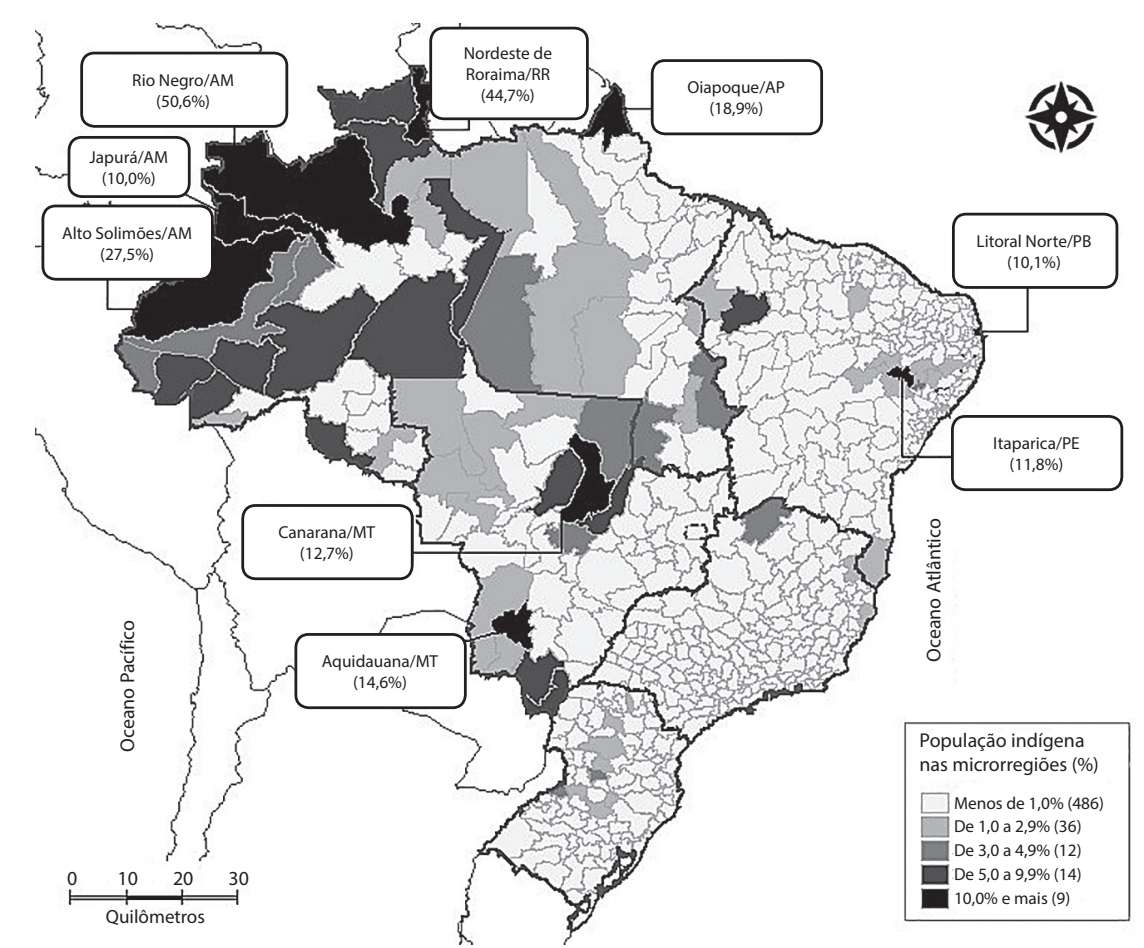

Fonte: Censo Demográfico 2010.

Figura 1 - População indígena residente nas microrregiões do Brasil com destaque para as nove unidades onde os indígenas representavam 10,0\% ou mais do total da população, 2010 
Tabela 1 - Frequências de óbitos infantis e de crianças menores de um ano indígenas e não indígenas de acordo com a distribuição proporcional de indígenas nas microrregiões, Brasil, 2010

\begin{tabular}{|c|c|c|c|c|c|c|c|}
\hline \multirow{2}{*}{$\begin{array}{l}\text { Proporção de } \\
\text { indígenas nas } \\
\text { microrregiões }\end{array}$} & \multirow[b]{2}{*}{$\mathbf{n}$} & \multicolumn{2}{|c|}{ Indígenas } & \multicolumn{2}{|c|}{ Não Indígenas } & \multicolumn{2}{|c|}{ Total } \\
\hline & & Óbitos infantis & $\begin{array}{c}\text { Crianças } \\
<1 \text { ano idade }\end{array}$ & Óbitos infantis & $\begin{array}{c}\text { Crianças } \\
<1 \text { ano idade }\end{array}$ & Óbitos infantis & $\begin{array}{c}\text { Crianças } \\
<1 \text { ano idade }\end{array}$ \\
\hline$\geq$ a $10,0 \%$ & 9 & 141 & 6.122 & 220 & 13.922 & 361 & 20.044 \\
\hline De 5,0 a $9,9 \%$ & 14 & 109 & 5.117 & 627 & 38.664 & 736 & 43.781 \\
\hline De 3,0 a $4,9 \%$ & 12 & 31 & 1.723 & 423 & 26.581 & 454 & 28.304 \\
\hline De 1,0 a $2,9 \%$ & 36 & 69 & 3.318 & 1.726 & 124.582 & 1.795 & 127.900 \\
\hline$<$ de $1,0 \%$ & 487 & 120 & 6.567 & 31.589 & 2.485 .350 & 31.709 & 2.491 .917 \\
\hline Total & 558 & 470 & 22.847 & 34.585 & 2.689 .099 & 35.055 & 2.711 .946 \\
\hline
\end{tabular}

Fonte: Censo Demográfico 2010

Para as 558 microrregiões foram registrados, em média, 62,9 óbitos infantis (variando de 2 a 1.985 óbitos). Houve, em média, 0,8 mortes de indígenas menores de um ano por microrregião (variando de 0 a 75 óbitos), sendo que em 124 microrregiões (22,2\% do total) houve pelo menos um óbito. Por outro lado, a maioria dos óbitos (53,2\%) ocorreu em apenas 23 microrregiões $(4,7 \%)$, sendo que nelas os indígenas representavam pelo menos $5,0 \%$ do total da população. Nas microrregiões onde os indígenas representavam pelo menos $10 \%$ da população total, ocorreram quase dos óbitos infantis indígenas (Tabela 1 e Figura 1).

No Brasil, como um todo, as mortes de crianças menores de um ano foram $60 \%$ mais frequentes entre as indígenas, comparadas às demais crianças (Razão de TMI $=1,60 ; p<0,01$ ). As probabilidades de morte de crianças menores de um ano foram mais expressivas para os domicílios indígenas em relação aos não indígenas em todos os estratos de microrregiões, variando de 21,1 por mil nascidos vivos (12 microrregiões onde 3,0 a 4,9\% da população era indígena) a 26,9 por mil (9 microrregiões com $10 \%$ ou mais de indígenas) (Figura 2).

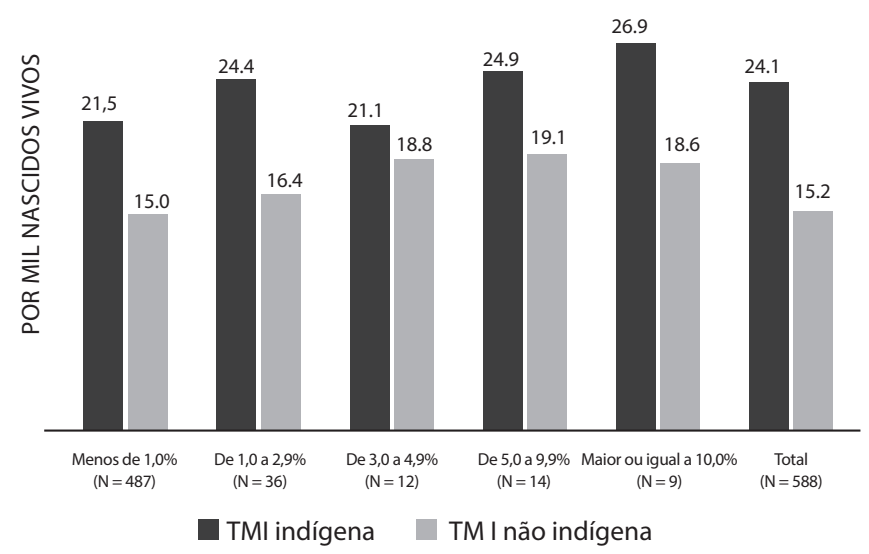

Fonte: Censo Demográfico 2010.

Figura 2 - Taxas de Mortalidade Infantil indígena e não indígena segundo distribuição proporcional de indígenas nas microrregiões, Brasil, 2010

Considerando as nove microrregiões com $10 \%$ ou mais de indígenas, observa-se que, com a exceção da microrregião Litoral Norte, localizada no extremo Leste do Nordeste brasileiro, em todas as demais a probabilidade de morte antes de completar um ano de vida foi maior para crianças indígenas em relação às não indígenas (Tabela 2). A magnitude mais expressiva na comparação dos níveis de mortalidade de crianças foi observada na microrregião do Alto Solimões (AM), onde mais de $1 / 4$ da população era indígena. $O$ risco de morte de crianças indígenas nessa microrregião foi $77 \%$ superior ao valor registrado para crianças não indígenas (Tabela 2).

Tabela 2 - Razões entre Taxas de Mortalidade Infantil indígena (A) e não indígena (B) nas nove microrregiões do Brasil, onde os indígenas representavam, pelo menos, 10,0\% do total de habitantes, Brasil, 2010

\begin{tabular}{lcccc}
\hline Microrregião - UF & $\begin{array}{c}\text { População } \\
\text { indígena } \\
\text { (\% do total) }\end{array}$ & $\begin{array}{c}\text { TMI } \\
\text { indígena } \\
\text { (A) }\end{array}$ & $\begin{array}{c}\text { TMI não } \\
\text { indígena } \\
\text { (B) }\end{array}$ & $\begin{array}{c}\text { Razão } \\
\text { de Taxas } \\
\text { (A/B) }\end{array}$ \\
\hline Rio Negro - AM & 50,6 & 15,9 & 15,2 & 1,05 \\
Nordeste de Roraima - RR & 44,7 & 14,6 & 13,4 & 1,09 \\
Alto Solimões - AM & 27,6 & 34,3 & 19,3 & 1,77 \\
Oiapoque - AP & 18,9 & 19,2 & 15,7 & 1,22 \\
Aquidauana - MS & 14,6 & 18,2 & 14,5 & 1,26 \\
Canarana - MT & 12,7 & 19,1 & 14,1 & 1,36 \\
Itaparica - PE & 11,8 & 18,4 & 14,0 & 1,32 \\
Litoral Norte - PB & 10,1 & 6,4 & 9,9 & 0,65 \\
Japurá - AM & 10,0 & 27,8 & 23,5 & 1,18 \\
\hline Fonte: Censo Demográfico 2010. & & & &
\end{tabular}

Convém ressaltar que os resultados descritos na Tabela 2 visam comparar as Taxas de Mortalidade Infantil entre indígenas e não indígenas, destacando as diferenças através da razão entre os valores registrados nas microrregiões selecionadas. Por serem dados oriundos do universo do Censo Demográfico 2010, os resultados não contêm erros amostrais, ainda que estejam sujeitos a variação aleatória, particularmente nas regiões de menor população.

A Tabela 3 apresenta, para as microrregiões com $10 \%$ ou mais de indígenas, a distribuição proporcional dos óbitos de acordo com as faixas de idade das mortes e os Capítulos da CID-10. Nota-se que a maioria dos óbitos de crianças indígenas $(67,7 \%)$ acometeu crianças no período pós-neonatal (29 a 364 dias), enquanto que para não indígenas a maior concentração foi no período de 0-6 dias (52,2\%) (Tabela 3). Em relação às causas, 34,2\% dos óbitos de crianças indígenas decorreram de doenças infecciosas, parasitárias e respiratórias, valor expressivamente mais pronunciado que o observado para as crianças não indígenas (12,3\%), cuja maioria das mortes ocorreu devido a afecções perinatais (Tabela 3). 
Tabela 3 - Mortalidade proporcional de crianças indígenas e não indígenas, segundo capítulos da CID-10, de acordo com o conjunto de microrregiões onde os indígenas representavam pelo menos 10,0\% do total de habitantes $(n=9)$, Brasil, 2010

\begin{tabular}{|c|c|c|c|}
\hline & & $\begin{array}{c}\text { Indígenas } \\
\text { (\%) } \\
\mathbf{n}=158\end{array}$ & $\begin{array}{c}\text { Não } \\
\text { indígenas } \\
(\%) \\
\mathbf{n = 2 0 3}\end{array}$ \\
\hline 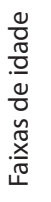 & $\begin{array}{l}0 \text { a } 6 \text { dias } \\
7 \text { a } 27 \text { dias } \\
28 \text { a } 364 \text { dias } \\
\text { Total }\end{array}$ & $\begin{array}{c}22,2 \\
10,1 \\
67,7 \\
100,0\end{array}$ & $\begin{array}{c}52,2 \\
9,4 \\
38,4 \\
100,0\end{array}$ \\
\hline $\begin{array}{l}\stackrel{ }{1} \\
\stackrel{1}{U} \\
\frac{0}{3} \\
\frac{\mathbb{Z}}{2} \\
\frac{0}{0}\end{array}$ & $\begin{array}{l}\text { I. Algumas doenças infecciosas e parasitárias } \\
\text { IV. Doenças endócrinas nutricionais e metabólicas } \\
\text { VI. Doenças do sistema nervoso } \\
\text { X. Doenças do aparelho respiratório } \\
\text { XVI. Algumas afecções originadas no período perinatal } \\
\text { XVII. Malf. cong. e formid. e anomalias cromossômicas } \\
\text { XVIII. Sint sinais e achad. Anorm. ex. clín. e laborat } \\
\text { Outras causas (ref. a demais capítulos) } \\
\text { Total }\end{array}$ & $\begin{array}{c}17,1 \\
8,9 \\
2,5 \\
17,1 \\
29,7 \\
6,3 \\
18,4 \\
0,0 \\
100,0\end{array}$ & $\begin{array}{c}7,4 \\
3,4 \\
0,0 \\
4,9 \\
51,2 \\
18,2 \\
8,4 \\
6,4 \\
100,0\end{array}$ \\
\hline
\end{tabular}

\section{DISCUSSÃO}

A mortalidade de crianças menores de um ano é um dos aspectos mais importantes para caracterizar as condições de vida e saúde às quais estão submetidas uma determinada população(1-2). Em âmbito global, a redução das Taxas de Mortalidade Infantil nos próximos anos configura uma das metas a serem alcançadas em países onde os indicadores socioeconômicos remetem a condições menos favoráveis ${ }^{(1)}$. Para os povos indígenas esse tem sido, indubitavelmente, um marcador que evidencia as enormes disparidades existentes em relação às crianças não indígenas no Brasil, apontando para a necessidade de maiores investimentos em setores sociais básicos (saúde, educação, habitação, saneamento, etc.) ${ }^{(14-15)}$.

Os resultados aqui descritos se alinham com aqueles observados em diversos outros estudos que apontaram elevadas magnitudes da morte de crianças indígenas, destacando perfil marcadamente distinto daquele observado para os não indígenas, o que se observa não somente nos níveis expressos pelas taxas, como também na distribuição de acordo com a idade e a causa de óbito(3,5,14-15).

No Brasil, ao longo das últimas décadas, se observou uma expressiva diminuição dos níveis da mortalidade infantil, tanto no país como um todo, quanto em contextos e localidades específicas $^{(2,16)}$. Entre 2000 e 2010, a Taxa de Mortalidade Infantil (TMI) caiu de 29,7 para 15,6 óbitos a cada mil nascidos vivos, enquanto que para crianças indígenas a redução foi de 74,6 para 41,9 óbitos a cada mil nascidos vivos ${ }^{(5)}$. Ou seja, a queda da TMl em indígenas no período não foi somente proporcionalmente menor, como também a magnitude se manteve expressivamente mais elevada.

Atribui-se que a redução da mortalidade infantil no Brasil ocorreu devido a uma conjugação de fatores socioeconômicos, demográficos e epidemiológicos, incluindo aumento da escolaridade materna, redução da fecundidade, diminuição da carga de doenças infecto-parasitárias, entre outras ${ }^{(2,9,16)}$. Não obstante, é importante reconhecer que persistem no país expressivas desigualdades nos níveis de mortalidade infantil, que se expressam tanto entre as regiões do país (níveis mais elevados no Norte e menores no Sul e Sudeste), como em relação aos estratos socioeconômicos ${ }^{(2,16)}$.

No âmbito dos debates sobre determinantes sociais em saúde no Brasil, há uma crescente ênfase em análises que levam em consideração a dimensão do pertencimento étnico-racial(4). No que diz respeito à mortalidade infantil, diversas análises têm evidenciado maiores níveis em crianças de cor ou raça preta e parda, em comparação às brancas e amarelas ${ }^{(3-4,6)}$, ao mesmo tempo que poucos estudos abordaram os diferenciais entre indígenas e não indígenas ${ }^{(5)}$. Desde os anos 1990, tem ocorrido importante expansão dos estudos sobre a saúde da população indígena no Brasil, buscando-se reverter o que já foi denominada de uma "danosa invisibilidade demográfica e epidemiológica"(17). Nesse contexto, o tema da mortalidade infantil tem recebido atenção, evidenciando-se que os níveis para indígenas, em geral, se mostram inclusive mais pronunciados que aqueles das demais categorias de cor ou raça $a^{(3,5)}$.

$\mathrm{O}$ risco de morte mais acentuado para crianças indígenas menores de um ano se expressa também em contextos locais. De acordo com a SESAI (Secretaria Especial de Saúde Indígena - ligada ao Ministério da Saúde), entre 2000 e 2012, registrou-se uma taxa média de 116,5 óbitos a cada mil nascidos vivos no DSEI Yanomami e de 94,7 óbitos por mil nascidos vivos no DSEI Xavante. Ainda que as TMI tenham se apresentado de maneira heterogênea nos diversos DSEI (em 2012 variou de 37,9 no DSEI Médio Rio Tapajós a 116,5 óbitos por mil nascidos vivos no DSEI Yanomami - ambos na Amazônia Legal), as taxas observadas são inequivocamente mais elevadas do que aquelas registradas para a população não indígena*.

A respeito das proporções de mortes de crianças de acordo com as faixas de idade ( 0 a 6 dias, 7 a 28 dias e 29 a 364 dias) descritas neste estudo, chama atenção a inversão das tendências entre as mortes ocorridas no período neonatal e pós-neonatal, quando comparadas as crianças indígenas e não indígenas. No período acima de um mês de vida, denominado "pós-neonatal", observou-se que a frequência de óbitos indígenas foi quase o dobro de não indígenas (67,7\% e 38,4\%, respectivamente). Além disso, as mortes de indígenas nas localidades analisadas (microrregiões onde pelo menos $10 \%$ da população era indígena) foram ocasionadas principalmente por agravos preveníveis (infecções parasitárias, respiratórias e desnutrição) (Tabela 3).

Diante da escassez de informações epidemiológicas específica para os indígenas, uma importante iniciativa foi a realização do I Inquérito Nacional de Saúde e Nutrição dos Povos Indígenas $(2008-2009)^{(15)}$. Os resultados dessa pesquisa revelaram que crianças indígenas das regiões Norte e Centro-Oeste apresentaram as

* As informações são oriundas do Sistema de Informação da Atenção à Saúde Indígena (SIASI), cujas tabulações podem ser realizadas em ambiente virtual da Secretaria Especial de Saúde Indígena (SESAI/Ministério da Saúde) através do endereço eletrônico http://portalsaude.saude.gov.br/index.php/o-ministerio/ principal/secretarias/secretaria-sesai. Acesso em 21 de junho de 2015. 
maiores prevalências tanto de desnutrição (40,8\% e 27,6\%, respectivamente) quanto de anemia (66,4\% e 51,5\%, respectivamente). Para as crianças não indígenas, levantamentos contemporâneos realizados no Brasil indicaram prevalência global de desnutrição da ordem de $7,1 \%$, sendo que na região Norte, esse indicador foi o dobro da média nacional $(14,7 \%)^{(15)}$.

O cenário de saúde das crianças e jovens indígenas da Amazônia é extremamente complexo, caracterizado por uma importante transição em saúde, na qual se observa uma sobreposição de doenças infecciosas e carências nutricionais, agravos crônicos não transmissíveis e causas externas (particularmente, suicídios) no quadro de morbimortalidade. Além disso, a situação de saúde das crianças indígenas se configura como bastante distinta daquela observada para as não indígenas, incluindo, entre outros aspectos, níveis substancialmente mais elevados de mortalidade infantil, de déficits nutricionais e de ocorrência de doenças infecciosas ligadas às condições de vida, com destaque para ausência de saneamento adequado.

\section{Limitações do estudo}

A magnitude da mortalidade infantil indígena foi analisada através de taxas calculadas a partir dos dados do recenseamento nacional realizado no Brasil, em 2010. Comparações de valores absolutos registrados pelo Censo e pelo SIM devem ser cautelosas devido às particularidades metodológicas empregadas em cada uma das fontes ${ }^{(5)}$.

As análises buscaram identificar desigualdades na ocorrência deste evento em relação ao grupo de crianças "não indígenas", o que limita comparações com os demais grupos de cor ou raça (branca, preta, amarela e parda), bem como o estudo de desigualdades entre esses segmentos da população. Por não serem realizadas análises em escala estadual, optou-se nesse trabalho por não empregar os fatores de correção de óbitos e nascidos vivos utilizados em análises recentes sobre mortalidade infantil segundo recorte de cor ou raça(5).

Há diversas outras possibilidades de aprofundamentos analíticos, a partir de dados censitários, inclusive para etnias específicas, e contextos geográficos específicos, como é o caso das terras indígenas, o que se pretende investigar em análises futuras.

\section{Contribuições para a área da Enfermagem, Saúde ou Política Pública}

Avaliações recentes têm apontado que o subsistema de Atenção à Saúde Indígena tem avançado em sua implantação, ao mesmo tempo em que se observam a permanência e o agravamento de problemas em sua operacionalização( ${ }^{(9)}$. Conforme indicado por Cardoso et al., (p. 930) "o modelo está longe de funcionar a contento, o que remete a dificuldades de várias ordens, incluindo a gerência, os recursos humanos e a articulação com os demais níveis do SUS"(9).

Nos 34 DSEl existentes no país, há uma expressiva presença de profissionais de enfermagem ${ }^{(15,17)}$. Em decorrência, é fundamental que se invista na disseminação de informações sobre os cenários epidemiológicos e a sociodiversidade dos povos indígenas no Brasil, tendo os profissionais de enfermagem como público-alvo. Cabe indicar também que tem sido cada vez mais frequente a formação de profissionais de enfermagem indígenas ${ }^{(17)}$.

\section{CONCLUSÃO}

O estudo da mortalidade infantil conduzido a partir da seleção de populações de diferentes pertencimentos étnico/raciais e que residiam em contextos espaciais específicos sugere um cenário de expressivas iniquidades em saúde, no qual os indígenas apresentaram maiores desvantagens. As comparações da mortalidade infantil considerando os volumes populacionais de indígenas demonstrou, sistematicamente, riscos mais elevados de morte para crianças indígenas. Nos contextos geográficos nos quais os indígenas representavam pelo menos $10 \%$ da população total, chama atenção que o risco de morte de crianças indígenas foi cerca de $40 \%$ mais elevado do que para não indígenas.

Há uma expressiva sociodiversidade indígena no Brasil, que vem sendo cada vez mais investigada no âmbito dos recenseamentos nacionais, contemplando uma extensa variedade de contextos geográficos. Os censos decenais vêm se tornando fonte de dados representativa dos segmentos populacionais minoritários, o que colabora para que indicadores gerados a partir de fontes governamentais se tornem ferramentas para elaboração de políticas públicas equitativas.

Este estudo caracteriza-se como uma iniciativa inédita ao analisar a ocorrência de óbitos de crianças indígenas em conformações territoriais específicas, chamando a atenção para contextos com maiores volumes populacionais indígenas.

\section{FOMENTO}

A pesquisa contou com apoio financeiro da FAPERJ (E26/102.352/2013) e do CNPq (304358/2014-2).

\section{REFERÊNCIAS}

1. UNICEF, WHO, World Bank, UN-DESA Population Division. Levels and Trends in Child Mortality 2015. Geneva: World Health Organization; 2015 [cited 2018 Nov 3]. 36 p. http://www.who.int/maternal_child_adolescent/documents/levels_trends_child_mortality_2015/en/\#. WgUGFo_chgY.mendeley.

2. Victora CG, Aquino EML, do Carmo Leal M, Monteiro CA, Barros FC, Szwarcwald CL. Maternal and child health in Brazil: progress and challenges. Lancet. 2011;377(9780):1863-76. doi:10.1016/S0140-6736(11)60138-4

3. Cardoso AM, Santos RV, Coimbra Jr. CEA. [Infant mortality according to race/color in Brazil: what do the national databases say?] Cad Saude Publica [Internet]. 2005 [cited 2018 Nov 02];21(5):1602-1608. Available from: doi: https://doi.org/10.1590/S0102-311X2005000500035 Portuguese 
4. Chor D. [Health inequalities in Brazil: race matters]. Cad Saude Publica [Internet]. 2013 [cited 2018 Nov 02];29(7):1272-1275. Avaliable from: https://doi.org/10.1590/S0102-311X2013000700002

5. Caldas ADR, Santos RV, Borges GM, Valente JG, Portela MC, [Infant mortality according to color or race based on the 2010 population census and national health information systems in Brazil]. Cad Saude Publica [Internet]. 2017 [cited 2018 Nov 02];33(7):e00046516. Available from: https://doi.org/10.1590/0102-311x00046516 Portuguese

6. Wong LLR, Sánchez JA (Orgs.). La Población Afro Descendiente e Indígena en América Latina: puntos de reflexión para el debate sobre Cairo + 20. Belo Horizonte: ALAP; 2014 [cited 2018 Nov 3]. 254 p. Available from: https://www.uaeh.edu.mx/investigacion/productos/6944/ capitulo_del_libro_los_nuevos_escenarios_de_la_migracion_pdf.pdf. Accessed November 10, 2017

7. Campos MB, Borges GM, Queiroz BL, Santos RV. [Differences in mortality between indigenous and non-indigenous persons in Brazil based on the 2010 Population Census]. Cad Saude Publica [Internet]. 2017 [cited 2018 Nov 02]; 33(5):e00015017. Available from: https://doi. org/10.1590/0102-311X00015017 Portuguese.

8. Instituto Brasileiro de Geografia e Estatística (IBGE). Características Gerais dos Indígenas: resultados do universo [Internet]. Rio de Janeiro: IBGE; 2010 [cited 2018 Nov 3] Available from: https://ww2.ibge.gov.br/home/estatistica/populacao/censo2010/caracteristicas_gerais_ indigenas/default_caracteristicas_gerais_indigenas.shtm

9. Giovanella L, Escorel S, Lobato LV, de Noronha JC, de Carvalho, Al (Orgs.). Políticas e Sistemas de Saúde no Brasil. In: Cardoso AM, Santos, RV, Garnelo, L, Coimbra Jr CEA, Chaves, MBG. Políticas Públicas de Saúde para os Povos Indígenas. 2 ed. Rio de Janeiro: FioCruz, Centro Brasileiro de Estudos de Saúde; 2012. p.911-32.

10. Queiroz BL, Sawyer DOT. O que os dados de mortalidade do Censo de 2010 podem nos dizer? R Bras Estud Popul [Internet]. 2012 [cited 2018 Nov 02];29(2):225-238. Available from: http://www.scielo.br/pdf/rbepop/v29n2/a02v29n2 doi:10.1590/S0102-30982012000200002

11. Wachter KW. Essential Demographic Methods. Harvard University Press; 2014. 288 p.

12. Keyfitz N, Flieger W. World Population: An Analysis of Vital Data. The University of Chicago Press; 1970. 672 p.

13. Anderson I, Robson B, Connolly M, et al. Indigenous and tribal peoples' health (The Lancet-Lowitja Institute Global collaboration): a population study. Lancet. 2016;388(10040):131-57. doi: 10.1016/S0140-6736(16)00345-7

14. Coimbra CE Jr, Santos RV, Welch JR, et al. The first national survey of indigenous people's health and nutrition in Brazil: rationale, methodology, and overview of results. BMC Public Health. 2013;13:52. doi:10.1186/1471-2458-13-52

15. Nascimento SG do, Oliveira CM de, Sposito V, et al. [Infant mortality due to avoidable causes in a city in Northeastern Brazil]. Rev Bras Enferm [Internet]. 2014 [cited 2018 Nov 02];67(2):208-12. Available from: https://doi.org/10.5935/0034-7167.20140027 Portuguese.

16. Coimbra Jr. CEA, Santos RV. [Health, minorities and inequality: some webs of inter-relations, emphasizing indigenous peoples in Brazil]. Cien Saude Colet [Internet]. 2000 [cited 2018 Nov 02];5(1):125-32. Available from: https://doi.org/10.1590/S1413-81232000000100011 Portuguese

17. Teixeira CC, Garnelo L (Orgs.). Saúde Indígena Em Perspectiva : Explorando Suas Matrizes Históricas e Ideológicas. In: Dias da Silva, C. De improvisos e cuidados: a saúde indígena e o campo da enfermagem. Rio de Janeiro: FIOCRUZ; 2014. p.181-212. 